Myburgh, J.A., Finfer, S., Bellomo, R., Billot, L., Cass, A., Gattas, D., Glass, P., Lipman, J., Liu, B., McArthur, C., McGuinness, S., Rajbhandari, D., Taylor, C.B. \& Webb, S.A.R. (2012). Hydroxyethyl Starch or Saline for Fluid Resuscitation in Intensive Care. New England J ournal of Medicine, 367, 1901-1911.

Copyright @ (2012) Massachusetts Medical Society. Reprinted with permission.

This is the final published version of the article accepted for publication in the New England J ournal of Medicine, following peer review. The definitive published version (see citation above) is located on the article abstract page of the publisher, Massachusetts Medical Society.

This version was made available in the UWA Research Repository on $31^{\text {st }}$ October 2013, in compliance with the publisher's policies on archiving in institutional repositories. 
ORIGINAL ARTICLE

\section{Hydroxyethyl Starch or Saline for Fluid Resuscitation in Intensive Care}

\author{
John A. Myburgh, M.D., Ph.D., Simon Finfer, M.D., Rinaldo Bellomo, M.D., \\ Laurent Billot, M.Sc., Alan Cass, M.D., Ph.D., David Gattas, M.D., \\ Parisa Glass, Ph.D., Jeffrey Lipman, M.D., Bette Liu, Ph.D., Colin McArthur, M.D., \\ Shay McGuinness, M.D., Dorrilyn Rajbhandari, R.N., Colman B. Taylor, M.N.D., \\ and Steven A.R. Webb, M.D., Ph.D., for the CHEST Investigators \\ and the Australian and New Zealand Intensive Care Society Clinical Trials Group*
}

\section{A BSTRACT}

\section{BACKGROUND}

The safety and efficacy of hydroxyethyl starch (HES) for fluid resuscitation have not been fully evaluated, and adverse effects of HES on survival and renal function have been reported.

\section{METHODS}

We randomly assigned 7000 patients who had been admitted to an intensive care unit (ICU) in a 1:1 ratio to receive either 6\% HES with a molecular weight of $130 \mathrm{kD}$ and a molar substitution ratio of 0.4 (130/0.4, Voluven) in $0.9 \%$ sodium chloride or $0.9 \%$ sodium chloride (saline) for all fluid resuscitation until ICU discharge, death, or 90 days after randomization. The primary outcome was death within 90 days. Secondary outcomes included acute kidney injury and failure and treatment with renal-replacement therapy.

\section{RESULTS}

A total of 597 of 3315 patients (18.0\%) in the HES group and 566 of $3336(17.0 \%)$ in the saline group died (relative risk in the HES group, 1.06; 95\% confidence interval [CI], 0.96 to 1.18; $\mathrm{P}=0.26$ ). There was no significant difference in mortality in six predefined subgroups. Renal-replacement therapy was used in 235 of 3352 patients (7.0\%) in the HES group and 196 of 3375 (5.8\%) in the saline group (relative risk, $1.21 ; 95 \% \mathrm{CI}, 1.00$ to $1.45 ; \mathrm{P}=0.04)$. In the HES and saline groups, renal injury occurred in $34.6 \%$ and $38.0 \%$ of patients, respectively $(\mathrm{P}=0.005)$, and renal failure occurred in $10.4 \%$ and $9.2 \%$ of patients, respectively $(\mathrm{P}=0.12)$. HES was associated with significantly more adverse events $(5.3 \%$ vs. $2.8 \%, \mathrm{P}<0.001)$.

\section{CONCLUSIONS}

In patients in the ICU, there was no significant difference in 90-day mortality between patients resuscitated with $6 \%$ HES $(130 / 0.4)$ or saline. However, more patients who received resuscitation with HES were treated with renal-replacement therapy. (Funded by the National Health and Medical Research Council of Australia and others; CHEST ClinicalTrials.gov number, NCT00935168.)
From the George Institute for Global Health (J.A.M., S.F., R.B., L.B., A.C., D.G., P.G., B.L., C.B.T., S.A.R.W.), University of New South Wales (J.A.M., B.L.), St. George Hospital (J.A.M.), University of Sydney (J.A.M., S.F., A.C., D.G., C.B.T.), Royal North Shore Hospital (S.F.), and Royal Prince Alfred Hospital (D.G., D.R.), Sydney; University of Melbourne and Austin Hospital, Melbourne, VIC (R.B.), University of Queensland and Royal Brisbane and Women's Hospital, Brisbane, QLD (J.L.), and University of Western Australia and Royal Perth Hospital, Perth, WA (S.A.R.W.) - all in Australia; and Auckland City Hospital, Auckland, New Zealand (C.M., S.M.). Address reprint requests to Dr. Myburgh at the Division of Critical Care and Trauma, George Institute for Global Health, Level 13, 321 Kent St., Sydney, NSW 2000, Australia, or at jmyburgh@georgeinstitute.org.au.

*Investigators in the Crystalloid versus Hydroxyethyl Starch Trial (CHEST) are listed in the Supplementary Appendix, available at NEJM.org.

This article was published on October 17, 2012, at NEJM.org.

N Engl J Med 2012;367:1901-11. DOI: 10.1056/NEJMoal209759

Copyright $(2012$ Massachusetts Medical Society. 
T HE ADMINISTRATION OF INTRAVENOUS fluids to increase intravascular volume is a frequent intervention in the intensive care unit (ICU), but the choice of resuscitation fluid remains controversial.1,2 Globally, $0.9 \%$ sodium chloride (saline) is the most commonly used fluid, although colloids are administered as often as crystalloids, and hydroxyethyl starch (HES) is the most frequently used colloid. ${ }^{3}$

Several studies have questioned the safety of HES in critically ill patients, with particular concern that its use increases the risk of acute kidney injury. ${ }^{4,5}$ Most concern has focused on the use of concentrated HES solutions (10\%) with a molecular weight of more than $200 \mathrm{kD}$ and a molar substitution ratio (the number of hydroxyethyl groups per glucose molecule) of more than 0.5.4,6 Commonly used HES solutions have a lower concentration (6\%) with a molecular weight of $130 \mathrm{kD}$ and molar substitution ratios of 0.38 to 0.45 . These preparations are available in different crystalloid carrier solutions. The clinical effects of lower-molecular-weight HES solutions have not been fully evaluated either in large clinical trials or in the broad range of ICU patients to whom they are now commonly administered. ${ }^{7}$ Additional concern arose from a recent Scandinavian trial reporting that the use of $6 \%$ HES (130/0.42) significantly increased mortality in patients with severe sepsis and septic shock. ${ }^{8}$

We conducted a large-scale, randomized, controlled trial to evaluate the safety and efficacy of $6 \%$ HES (130/0.4) in $0.9 \%$ saline as compared with $0.9 \%$ saline alone for fluid resuscitation in a heterogeneous population of adult patients treated in the ICU.

\section{METHODS}

\section{STUDY OVERSIGHT}

The Crystalloid versus Hydroxyethyl Starch Trial (CHEST) was an investigator-initiated, multicenter, prospective, blinded, parallel-group, randomized, controlled trial conducted in 32 hospitals in Australia and New Zealand.

The study-management committee designed the trial. The trial was endorsed by the Australian and New Zealand Intensive Care Society Clinical Trials Group. The study protocol ${ }^{9}$ (available with the full text of this article at NEJM.org) was approved by the human research ethics committee at the Northern Sydney and Central Coast Area Health Service, the ethics committee at the University of Sydney, and by each participating institution. Written informed consent before randomization or delayed consent was obtained from each patient, legal surrogate, or institutional ethics committee.

The statistical analysis plan $^{10}$ was reported previously. Statistical analyses were conducted at the George Institute for Global Health. The authors vouch for the data and statistical analyses, made the decision to submit the manuscript for publication, and assume responsibility for the fidelity of the final report to the protocol.

The study was funded by a project grant from the National Health and Medical Research Council of Australia and by unrestricted grants from the New South Wales Ministry of Health and Fresenius Kabi, the manufacturer of Voluven. Funding agencies had no input into the design, conduct, data collection, statistical analysis, or writing of the manuscript. Fresenius Kabi supplied the study fluids and distributed them to participating sites.

Independent analysis of the concentration and degree of molar substitution of HES and the concentration of saline was obtained for a random sample of 20 bags with the use of gravimetric analysis and nuclear magnetic resonance spectroscopy (Chemical Analysis).

\section{PATIENTS}

Patients who were 18 years of age or older were assessed for eligibility on admission to the ICU. Patients who met the eligibility criteria were those whom the treating clinician judged to require fluid resuscitation, which was defined as the administration of a bolus of intravenous fluid over and above that required for maintenance or replacement fluids. Fluid was administered to correct hypovolemia at any time during the patient's ICU admission, as determined by the treating clinician and supported by at least one objective physiological criterion (Table S1A in the Supplementary Appendix, available at NEJM.org). The treating clinicians determined the initial and subsequent volumes and the rate of administration of resuscitation fluid, depending on clinical signs and the subsequent response to fluid administration.

Patients who had received more than $1000 \mathrm{ml}$ of HES before screening, those with impending or current dialysis-dependent renal failure, and those with evidence of intracranial hemorrhage on cranial computed tomography were excluded. 
A full list of exclusion criteria is provided in Table S1B in the Supplementary Appendix.

\section{STUDY RANDOMIZATION AND TREATMENT}

Study treatments were randomly assigned over an encrypted Web-based randomization system with the use of a minimization algorithm stratified according to institution and an admission diagnosis of trauma.

Patients were assigned to receive either $6 \%$ HES (130/0.4) in $0.9 \%$ saline (Voluven, Fresenius Kabi) or $0.9 \%$ saline in indistinguishable Freeflex $500-\mathrm{ml} \mathrm{bags}^{9}$ for all fluid resuscitation in the ICU until discharge, death, or 90 days after randomization.

In accordance with registration by the Therapeutic Goods Administration of Australia and the New Zealand Medicines and Medical Devices Safety Authority for 6\% HES (130/0.4), study fluid was administered to a maximum dose of $50 \mathrm{ml}$ per kilogram of body weight per day, followed by open-label $0.9 \%$ saline for the remainder of the 24-hour period.

Study fluid was stopped in patients who were treated with any mode of renal-replacement therapy. In these patients, treatment with saline was recommended, but any other fluid, apart from HES, was permitted. The administration of resuscitation fluids outside the ICU was not controlled.

All other aspects of patient care, including maintenance fluids and nutrition, cardiovascular monitoring, pharmacologic support, and respiratory and renal support, were conducted at the discretion of the treating clinicians.

\section{OUTCOME MEASURES}

The primary outcome measure was all-cause mortality 90 days after randomization.

Secondary outcomes within the 90-day followup period were the incidence of acute kidney injury, as defined with the use of a five-category scoring system to evaluate risk, injury, failure, loss, and end-stage kidney injury (RIFLE) ${ }^{11}$; the use of renal-replacement therapy; new organ failures for cardiovascular, respiratory, coagulation, and liver systems that were not present at baseline; duration of mechanical ventilation and renal-replacement therapy; and cause-specific mortality.

Using the RIFLE diagnostic criteria, we evaluated patients for being at risk for kidney dysfunction (RIFLE-R), for injury to the kidney
(RIFLE-I), and for failure of kidney function (RIFLE-F) on the basis of incremental increases in serum creatinine levels from baseline and decreases in urine output over specified time periods. Patients with a complete loss of kidney function for more than 4 weeks (RIFLE-L) and end-stage kidney disease (RIFLE-E) completed the diagnostic criteria (Table S2 in the Supplementary Appendix). Included in the analysis was any mode of renal-replacement therapy that was prescribed by the attending physician. New organ failures for cardiovascular, respiratory, coagulation, and liver systems that were not present at baseline were defined by a score of 3 or more on the Sequential Organ Failure Assessment (SOFA), ${ }^{12}$ which ranges from 0 to 4 for each of six organ systems, including neurologic and renal systems, with higher scores indicating more severe organ dysfunction (Table S3 in the Supplementary Appendix).

Tertiary outcomes were the duration of ICU stay and hospital admission and the rate of death in the ICU and hospital.

The primary outcome was also examined in six subgroup pairs on the basis of baseline characteristics: the presence or absence of diagnostic criteria for urine output for acute kidney injury (RIFLE-R and RIFLE-I categories), the presence or absence of sepsis at randomization,,$^{13}$ the presence or absence of trauma with or without traumatic brain injury, the score ( $<25$ vs. $\geq 25$ ) on the Acute Physiology and Chronic Health Evaluation (APACHE) $\mathrm{II}^{14}$ (ranging from 0 to 71, with higher scores indicating an increased risk of death), and receipt or nonreceipt of HES before randomization.

\section{DATA AND STUDY MANAGEMENT}

Trained research coordinators collected data at each site and entered them into a Web-based database. Data monitoring and source data verification were conducted according to a prespecified monitoring plan. An independent data and safety monitoring committee reviewed data from prespecified interim analyses after the enrollment of 2000 and 4000 patients.

\section{STATISTICAL ANALYSIS}

We determined that a sample size of 7000 patients would provide a power of $90 \%$ to detect an absolute difference of 3.5 percentage points in 90-day mortality on the basis of an estimated baseline mortality of $26 \%$ at an alpha level of 0.05 with an 
assumption of a $5 \%$ loss to follow-up. ${ }^{10}$ In addition, the study had a power of $90 \%$ with an alpha level of 0.05 to detect an absolute increase in the relative risk of acute kidney injury of 1.5 percentage points from a baseline incidence of $6 \% .{ }^{4}$

We conducted all analyses on an intention-totreat basis. Methods for the handling of missing data are outlined in the Supplementary Appendix. We compared binary outcomes using relative risks with $95 \%$ confidence intervals and chisquare tests. Continuous outcomes were compared with the use of mean differences and unpaired t-tests. Adjusted analyses were performed with the use of robust Poisson regression for binary outcomes and robust linear regression for continuous outcomes. Baseline covariates included the presence or absence of trauma, age, ICU admission source, APACHE II score, and baseline serum creatinine level. We compared survival times using log-rank tests and present these as Kaplan-Meier curves. Comparisons of fluid and physiological data averaged over the first 4 days were performed with the use of t-tests.

For the predefined subgroups, we assessed the primary outcome using methods similar to those used for the main analysis, and we assessed heterogeneity of treatment effects among subgroup pairs using the test for a common relative risk. We conducted post hoc analyses comparing changes in serum creatinine levels and urine output during the first 7 days in each group and in patients who satisfied the diagnostic criteria for RIFLE-R, RIFLE-I, and RIFLE-F categories of acute kidney injury. All analyses were conducted with the use of SAS statistical software, version 9.2 (SAS Institute).

RESULTS

\section{PATIENTS}

From December 2009 through January 2012, we enrolled 7000 patients in 32 adult medical-surgical ICUs in Australia and New Zealand, with 3500 patients assigned to receive 6\% HES (130/0.4) in $0.9 \%$ saline (HES group) and 3500 to receive $0.9 \%$ saline (saline group) (Fig. 1). The two groups of patients had similar characteristics at baseline (Table 1). The primary outcome was available for 3315 patients $(94.7 \%)$ in the HES group and 3336 patients (95.3\%) in the saline group.

\section{FLUID THERAPY AND TREATMENT EFFECTS}

Independent analysis of the random sample of study fluids reported the concentration of HES to be between $5.97 \%$ and $6.00 \%$, with a mean degree of molar substitution of 0.44 . The concentration of saline in the HES bags was between $0.92 \%$ and $0.93 \%$.

During the first 4 days, the HES group received significantly less study fluid than the saline group (mean $[ \pm S D]$ daily average, $526 \pm 425 \mathrm{ml}$ vs. $616 \pm 488 \mathrm{ml} ; \mathrm{P}<0.001$ ), with most of the volume administered in the first 24 hours (Fig. S1 in the Supplementary Appendix).

The HES group also received significantly less nonstudy fluid than the saline group $(851 \pm 675 \mathrm{ml}$ vs. $1115 \pm 993 \mathrm{ml}, \mathrm{P}<0.001$ ), resulting in a significantly lower positive net fluid balance $(921 \pm 1069 \mathrm{ml}$ vs. $982 \pm 1161 \mathrm{ml}, \mathrm{P}=0.03$ ) (Table S4 and Fig. $\mathrm{S} 1$ in the Supplementary Appendix).

During the first 4 days, the HES group received significantly more blood products than the saline group ( $78 \pm 250 \mathrm{ml}$ vs. $60 \pm 190 \mathrm{ml}, \mathrm{P}<0.001)$, and central venous pressure was significantly higher in the HES group $(11.3 \pm 4.8 \mathrm{~mm} \mathrm{Hg}$ vs. $10.4 \pm 4.4 \mathrm{~mm} \mathrm{Hg}, \mathrm{P}<0.001$ ) (Fig. S1 and S2 in the Supplementary Appendix). There were no significant between-group differences in heart rate, mean arterial pressure, and lactate levels during the first 4 days (Fig. S2 in the Supplementary Appendix).

\section{OUTCOMES}

In the HES group, 597 of 3315 patients (18.0\%) died within 90 days after randomization, as compared with 566 of 3336 patients (17.0\%) in the saline group (relative risk in the HES group, 1.06; $95 \%$ confidence interval [CI], 0.96 to 1.18 ; $\mathrm{P}=0.26$ ) (Table 2). There was no significant difference in the probability of survival between the HES group and the saline group during the 90 days after randomization ( $\mathrm{P}=0.27)$ (Fig. $2 \mathrm{~A}$ ). There was no significant heterogeneity in the effect of treatment on 90-day mortality in any of the predefined subgroups (Fig. 2B). Renal-replacement therapy was administered to 235 of 3352 patients $(7.0 \%)$ in the HES group and 196 of 3375 patients $(5.8 \%)$ in the saline group (relative risk, 1.21 ; $95 \% \mathrm{CI}, 1.00$ to $1.45 ; \mathrm{P}=0.04$ ).

The diagnostic criteria for being at risk for various stages of renal injury were as follows: renal dysfunction (RIFLE-R), 1788 of 3309 pa- 


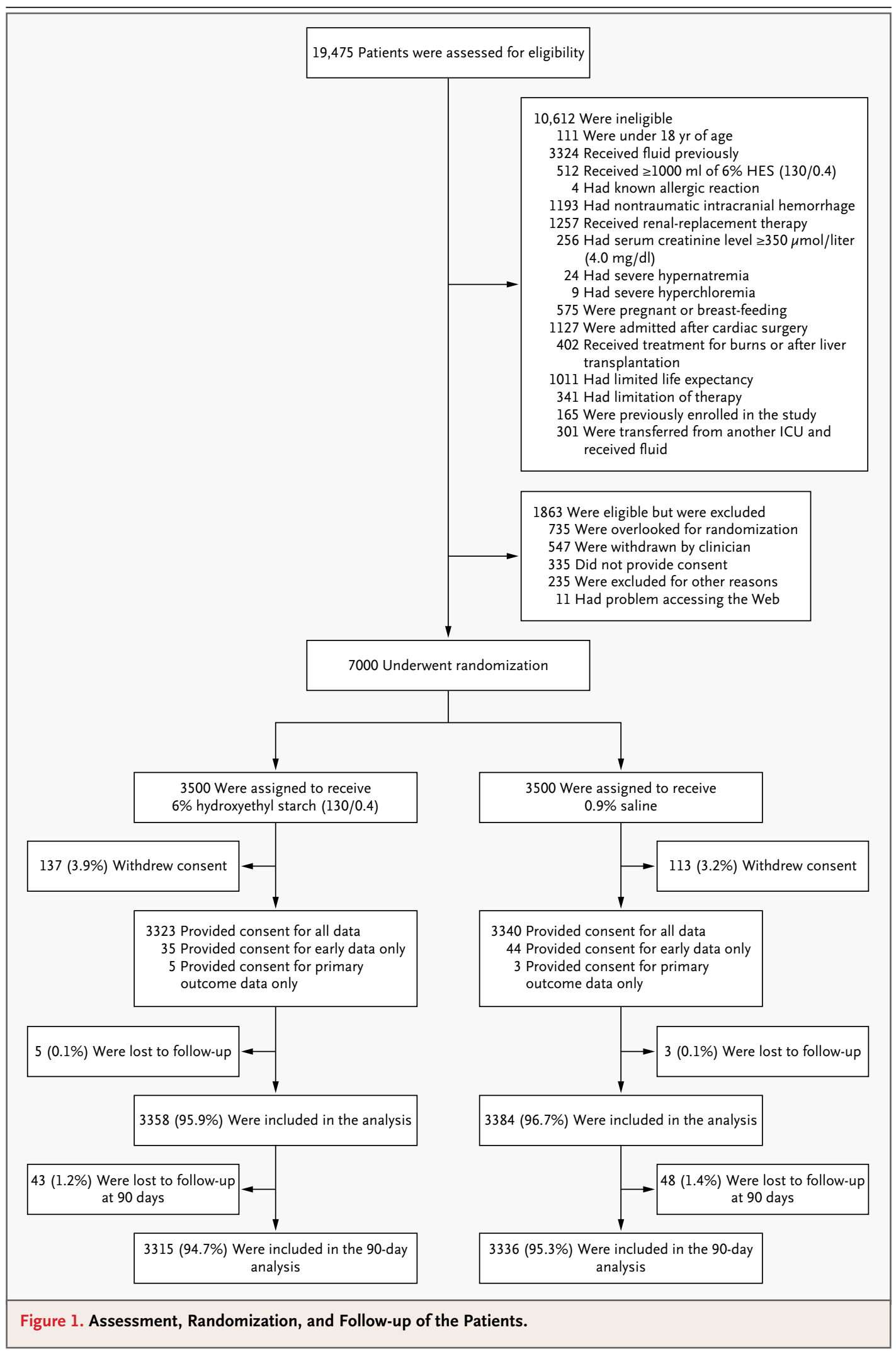

N ENGLJ MED 367;20 NEJM.ORG NOVEMBER 15, 2012

The New England Journal of Medicine

Downloaded from nejm.org at UNIVERSITY OF WESTERN AUSTRALIA on October 30, 2013. For personal use only. No other uses without permission. Copyright (C) 2012 Massachusetts Medical Society. All rights reserved. 


\begin{tabular}{|c|c|c|}
\hline Characteristic & $\begin{array}{c}\text { HES } \\
(\mathrm{N}=3358)\end{array}$ & $\begin{array}{c}\text { Saline } \\
(\mathrm{N}=3384)\end{array}$ \\
\hline Age $-y r$ & $63.1 \pm 17.0$ & $62.9 \pm 16.9$ \\
\hline Male sex — no./total no. (\%) & $2030 / 3356(60.5)$ & $2041 / 3384(60.3)$ \\
\hline Weight — kg & $79.4 \pm 21.0$ & $78.6 \pm 20.8$ \\
\hline \multicolumn{3}{|l|}{ Source of admission to ICU — no./total no. (\%) } \\
\hline Emergency department & $930 / 3353(27.7)$ & $931 / 3379(27.6)$ \\
\hline Hospital floor & $659 / 3353(19.7)$ & $668 / 3379(19.8)$ \\
\hline Another ICU & $53 / 3353(1.6)$ & $41 / 3379(1.2)$ \\
\hline Another hospital & $315 / 3353(9.4)$ & $306 / 3379(9.1)$ \\
\hline \multicolumn{3}{|l|}{ Operating room } \\
\hline After emergency surgery & $625 / 3353(18.6)$ & $630 / 3379(18.6)$ \\
\hline After elective surgery & $771 / 3353(23.0)$ & $803 / 3379(23.8)$ \\
\hline \multicolumn{3}{|l|}{ Diagnosis on admission — no./total no. (\%) } \\
\hline Surgical cases & $1426 / 3353(42.5)$ & $1450 / 3379(42.9)$ \\
\hline Nonsurgical cases & $1920 / 3353(57.3)$ & $1926 / 3379(57.0)$ \\
\hline APACHE II score - median (interquartile range) $\uparrow$ & $17.0(12.0-22.0)$ & $17.0(12.0-23.0)$ \\
\hline Time from ICU admission to randomization $-\mathrm{hr}$ & $10.9 \pm 156.5$ & $11.4 \pm 165.4$ \\
\hline \multicolumn{3}{|l|}{ Physiological variables } \\
\hline Heart rate - beats $/ \mathrm{min}$ & $89.3 \pm 23.6$ & $88.8 \pm 23.3$ \\
\hline Mean arterial pressure $-\mathrm{mm} \mathrm{Hg}$ & $74.0 \pm 14.9$ & $73.7 \pm 14.6$ \\
\hline Central venous pressure $-\mathrm{mm} \mathrm{Hg}$ & $9.5 \pm 5.4$ & $8.9 \pm 5.1$ \\
\hline Lactate $-\mathrm{mmol} / \mathrm{liter}$ & $2.1 \pm 2.0$ & $2.0 \pm 1.5$ \\
\hline Mechanical ventilation - no./total no. (\%) & $2131 / 3326(64.1)$ & $2177 / 3354(64.9)$ \\
\hline Use of vasopressor — no./total no. (\%) & $1520 / 3337(45.5)$ & $1551 / 3361(46.1)$ \\
\hline Serum creatinine $-\mu \mathrm{mol} /$ liter & $101.5 \pm 57.1$ & $100.1 \pm 58.0$ \\
\hline Urine output $6 \mathrm{hr}$ before randomization $-\mathrm{ml}$ & $453.5 \pm 418.3$ & $426.6 \pm 422.9$ \\
\hline \multicolumn{3}{|l|}{ Predefined subgroups — no./total no. (\%) } \\
\hline RIFLE criteria for acute kidney injury & $522 / 1449(36.0)$ & $511 / 1421(36.0)$ \\
\hline Sepsis & $979 / 3355(29.2)$ & $958 / 3376(28.4)$ \\
\hline Trauma & $267 / 3358(8.0)$ & $265 / 3384(7.8)$ \\
\hline Traumatic brain injury & $28 / 3338(0.8)$ & $30 / 3365(0.9)$ \\
\hline APACHE II score $\geq 25$ & $597 / 3335(17.9)$ & $624 / 3356(18.6)$ \\
\hline Receipt of HES before randomization & $509 / 3347(15.2)$ & $508 / 3372(15.1)$ \\
\hline
\end{tabular}

* Plus-minus values are means \pm SD. There were no significant differences between the groups except for central venous pressure $(P<0.001)$ and lactate level $(P<0.05)$. To convert the values for creatinine to milligrams per deciliter, divide by 88.4. HES denotes hydroxyethyl starch, and ICU intensive care unit.

$\uparrow$ Scores on the Acute Physiology and Chronic Health Evaluation (APACHE) II range from 0 to 71 , with higher scores indicating an increased risk of death.

$¥$ RIFLE (risk, injury, or failure) criteria for acute kidney injury at baseline were calculated from measures of available urine output only in patients who satisfied the criteria for risk and injury. Changes in serum creatinine levels were not applicable.

tients (54.0\%) in the HES group and 1912 of injury to the kidney (RIFLE-I), 1130 of 3265 pa3335 patients $(57.3 \%)$ in the saline group (rela- tients (34.6\%) in the HES group and 1253 of tive risk, $0.94 ; 95 \% \mathrm{CI}, 0.90$ to $0.98 ; \mathrm{P}=0.007) ; 3300$ patients $(38.0 \%)$ in the saline group (relative 


\begin{tabular}{|c|c|c|c|c|}
\hline Variable & HES & Saline & $\begin{array}{l}\text { Relative Risk } \\
\text { (95\% CI) }\end{array}$ & P Value \\
\hline \multicolumn{5}{|l|}{ Outcome } \\
\hline $\begin{array}{c}\text { Primary outcome of death at day } 90- \\
\text { no./total no. (\%) }\end{array}$ & $597 / 3315(18.0)$ & $566 / 3336(17.0)$ & $1.06(0.96$ to 1.18$)$ & 0.26 \\
\hline \multicolumn{5}{|l|}{ Secondary outcomes - no./total no. (\%) } \\
\hline \multicolumn{5}{|l|}{ Renal outcomes } \\
\hline RIFLE-R & $1788 / 3309(54.0)$ & $1912 / 3335(57.3)$ & 0.94 (0.90 to 0.98$)$ & 0.007 \\
\hline RIFLE-I & $1130 / 3265(34.6)$ & $1253 / 3300(38.0)$ & 0.91 (0.85 to 0.97$)$ & 0.005 \\
\hline RIFLE-F & $336 / 3243(10.4)$ & $301 / 3263(9.2)$ & $1.12(0.97$ to 1.30$)$ & 0.12 \\
\hline Use of renal-replacement therapy & $235 / 3352(7.0)$ & $196 / 3375(5.8)$ & 1.21 (1.00 to 1.45$)$ & 0.04 \\
\hline \multicolumn{5}{|l|}{ New organ failure $\dagger$} \\
\hline Respiratory & $540 / 2062(26.2)$ & $524 / 2094(25.0)$ & 1.05 (0.94 to 1.16$)$ & 0.39 \\
\hline Cardiovascular & $663 / 1815(36.5)$ & $722 / 1808$ (39.9) & 0.91 (0.84 to 0.99$)$ & 0.03 \\
\hline Coagulation & $142 / 2987(4.8)$ & $119 / 3010(4.0)$ & $1.20(0.95$ to 1.53$)$ & 0.13 \\
\hline Hepatic & $55 / 2830(1.9)$ & $36 / 2887(1.2)$ & 1.56 (1.03 to 2.36$)$ & 0.03 \\
\hline \multicolumn{5}{|l|}{ Tertiary outcomes - no./total no. (\%) } \\
\hline Death in ICU & $364 / 3313(11.0)$ & $360 / 3331(10.8)$ & 1.02 (0.89 to 1.17$)$ & 0.81 \\
\hline Death within 28 days & $458 / 3313(13.8)$ & $437 / 3331(13.1)$ & 1.05 (0.93 to 1.19$)$ & 0.40 \\
\hline \multirow[t]{2}{*}{ Death in hospital } & $483 / 3307(14.6)$ & $456 / 3324(13.7)$ & $1.06(0.95$ to 1.20$)$ & 0.30 \\
\hline & & \multicolumn{3}{|c|}{ Mean Difference $(95 \% \mathrm{Cl})$} \\
\hline \multicolumn{5}{|l|}{ Service utilization - no. } \\
\hline Days in ICU & $7.3 \pm 0.2$ & $6.9 \pm 0.2$ & $0.4(0.0$ to 0.9$)$ & 0.07 \\
\hline Days in hospital & $19.3 \pm 0.3$ & $19.1 \pm 0.3$ & $0.2(-0.8$ to 1.1$)$ & 0.72 \\
\hline Days receiving mechanical ventilation & $6.0 \pm 0.2$ & $5.7 \pm 0.2$ & $0.4(-0.1$ to 0.8$)$ & 0.12 \\
\hline Days receiving renal-replacement therapy & $5.6 \pm 0.4$ & $5.5 \pm 0.4$ & $0.1(-0.1$ to 1.2$)$ & 0.86 \\
\hline \multicolumn{5}{|l|}{ Treatment-related adverse events: } \\
\hline Any event - no./total no. (\%) & $180 / 3416(5.3)$ & $95 / 3358(2.8)$ & & $<0.001$ \\
\hline Pruritus & $137 / 3416(4.0)$ & $73 / 3358(2.2)$ & & \\
\hline Skin rash & $34 / 3416(1.0)$ & $16 / 3358(0.5)$ & & \\
\hline Other & $9 / 3416(0.3)$ & $6 / 3358(0.2)$ & & \\
\hline Serious adverse events - no./total no. (\%)』 & $2 / 3416(0.1)$ & $2 / 3358(0.1)$ & & 0.98 \\
\hline
\end{tabular}

risk, $0.91 ; 95 \% \mathrm{CI}, 0.85$ to $0.97 ; \mathrm{P}=0.005)$; and failure of kidney function (RIFLE-F), 336 of 3243 patients (10.4\%) in the HES group and 301 of 3263 patients $(9.2 \%)$ in the saline group (relative risk, $1.12 ; 95 \% \mathrm{CI}, 0.97$ to $1.30 ; \mathrm{P}=0.12$ ).

Post hoc analysis showed that serum creati- nine levels were significantly increased and urine output was significantly decreased in the HES group, as compared with the saline group, during the first 7 days $(\mathrm{P}=0.004$ and 0.003 , respectively) (Fig. 3). In addition, when the creatinine and urine-output components of each RIFLE catego- 


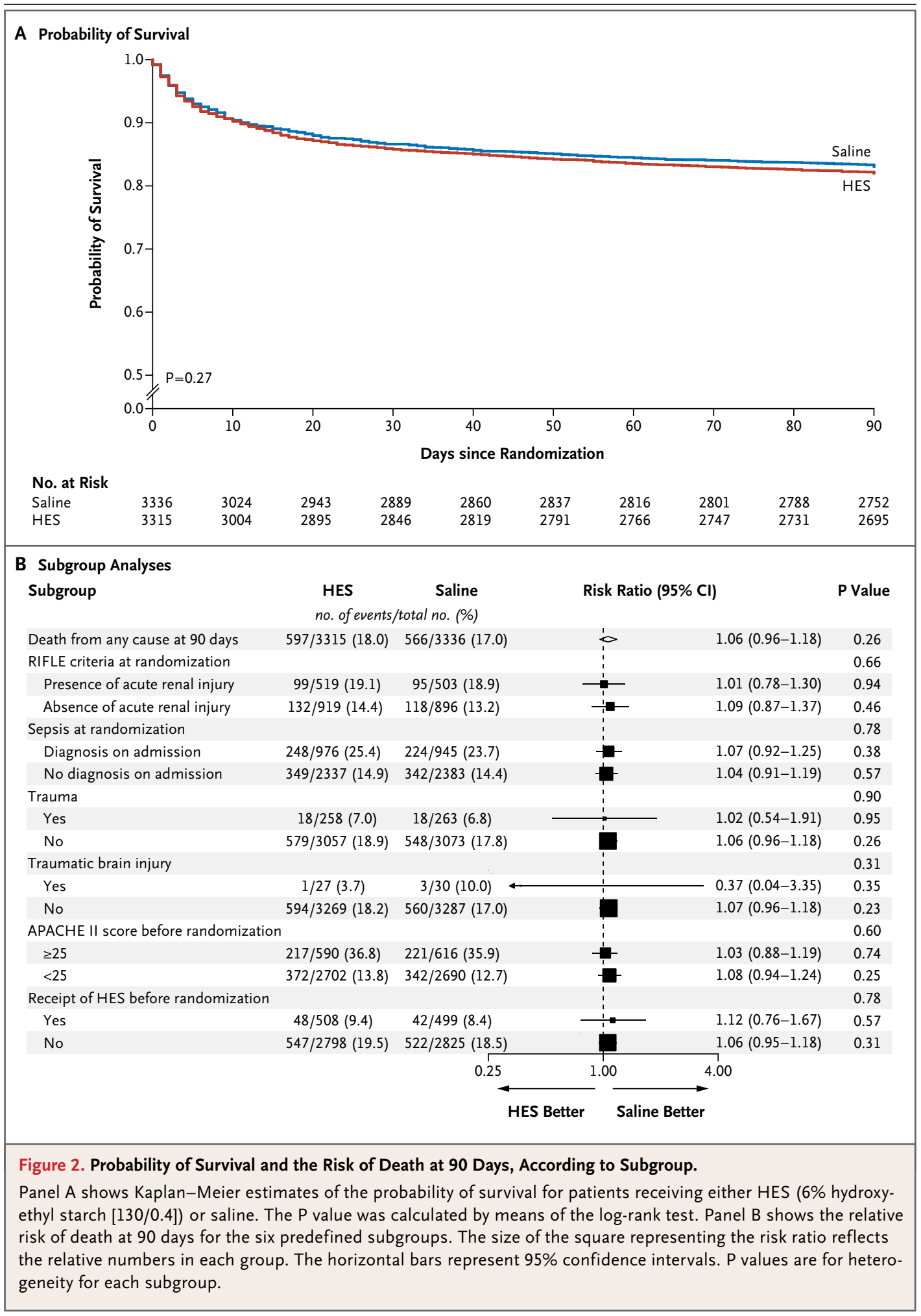

The New England Journal of Medicine 
ry were analyzed separately, the relative risks of meeting the criteria for the risk of kidney dysfunction (RIFLE-R) or kidney injury (RIFLE-I) were higher in the HES group than in the saline group (Fig. S3 in the Supplementary Appendix).

There was no significant between-group difference in rates of death in the ICU, at 28 days, and in the hospital or in rates of new respiratory or new coagulation organ failure. The incidence of new cardiovascular organ failure was significantly lower in the HES group than in the saline group (36.5\% vs. 39.9\%) (relative risk, $0.91 ; 95 \%$ CI, 0.84 to $0.99 ; \mathrm{P}=0.03$ ). The incidence of new hepatic organ failure was significantly higher in the HES group than in the saline group $(1.9 \%$ vs. $1.2 \%$ ) (relative risk, 1.56; 95\% CI, 1.03 to 2.36; $\mathrm{P}=0.03$ ). There were no significant differences in the duration of mechanical ventilation, of renal-replacement therapy, or of ICU or hospital stays between the two groups (Table 2). There was no significant between-group difference in cause-specific mortality within the 90day follow-up period (Table S5 in the Supplementary Appendix). The main results were similar after adjustment for baseline covariates (Table S6 in the Supplementary Appendix).

The use of HES was associated with a significant increase in the rate of adverse events (5.3 vs. $2.8 \%, \mathrm{P}<0.001$ ). Of these events, pruritus and rash were the most common (Table 2).

\section{DISCUSSION}

In this randomized, controlled trial, there was no significant difference in mortality at 90 days in a heterogeneous population of ICU patients who received $6 \%$ HES $(130 / 0.4)$ in $0.9 \%$ saline and those who received $0.9 \%$ saline alone for fluid resuscitation. The effect on mortality did not differ significantly in six predefined subgroup pairs.

Our study was a large-scale, pragmatic trial modeled on the Saline versus Albumin Fluid Evaluation (SAFE) trial. ${ }^{15}$ We sought to minimize the risk of bias through centralized randomization, allocation concealment, and blinding of studygroup assignments. We used a robust primary outcome measured at an interval relevant to this study population. ${ }^{16,17}$ To further minimize the risk of bias, we published the statistical analysis plan,

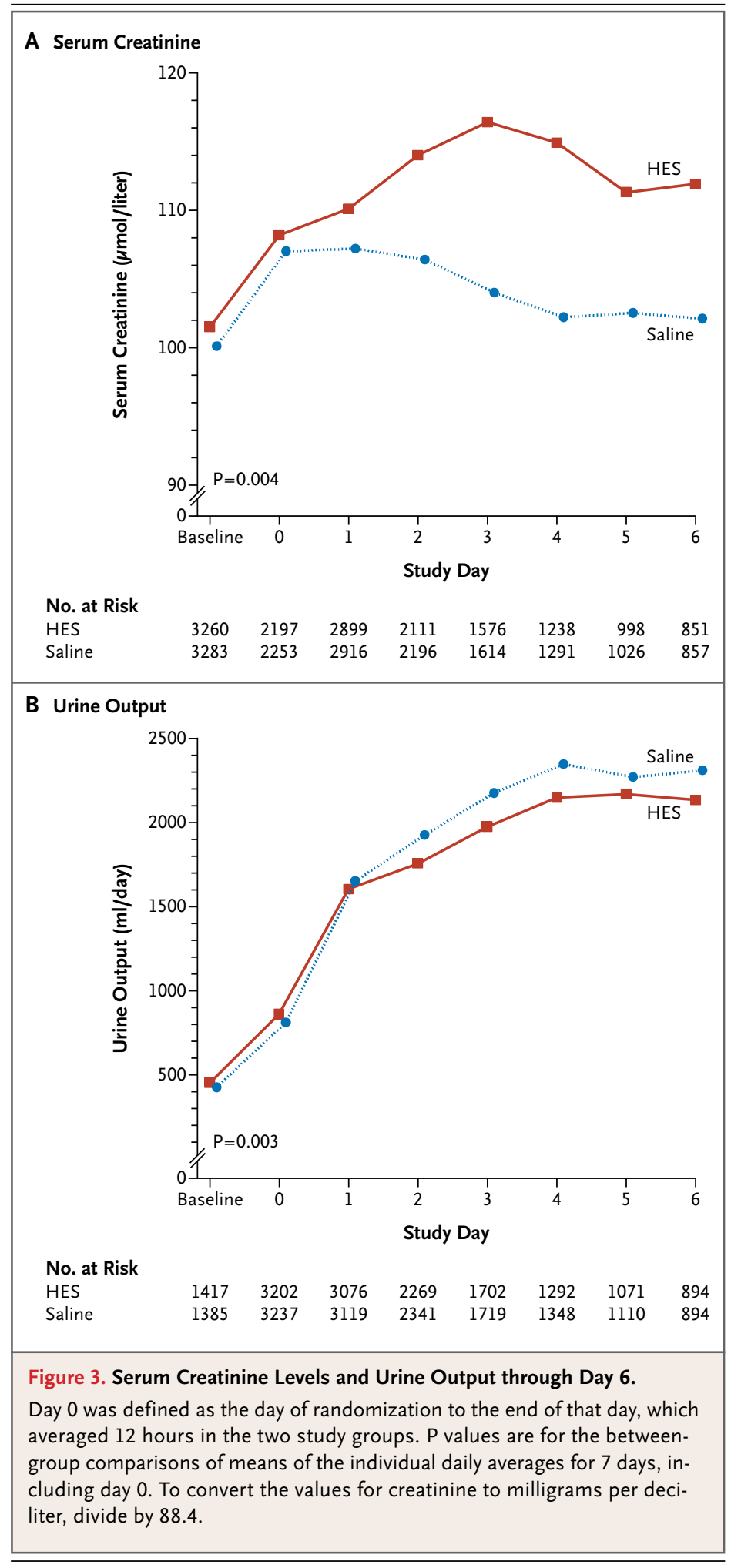


including predefined subgroups, before the unblinding of study-group assignments. ${ }^{10}$

A limitation of our study is that the observed rate of death was lower than predicted. This was due, in part, to the exclusion of patients with intracranial hemorrhage and those whom clinicians considered unlikely to survive, as well as the inclusion of patients who had undergone elective surgery. In addition, patients were recruited after admission to the ICU, when the requirements for fluid resuscitation are often less than those for patients in the emergency department or the operating room.

Despite these limitations, our trial had sufficient statistical power to detect an absolute mortality difference of 3 percentage points. Our results are consistent with a treatment effect that lies between a relative decrease of $4 \%$ or a relative increase of $18 \%$ in mortality arising from resuscitation with HES.

A fundamental reason for conducting our study was to determine whether currently used HES solutions increased the risk of acute kidney injury in ICU patients. Resuscitation with HES resulted in a $21 \%$ relative increase in the number of patients treated with renal-replacement therapy. Although criteria for the initiation of renalreplacement therapy were not outlined in the protocol and such therapy was initiated at the discretion of the attending clinicians, the clinicians were unaware of study-group assignments, which made it unlikely that the difference was caused by variations in thresholds for initiating therapy. ${ }^{18}$

The RIFLE score is a composite-end-point measure that combines effects on serum creatinine levels and urine output. In our study, the use of HES had opposite effects on the two components of this score, as compared with saline. Our post hoc analysis showed that treatment with HES was associated with increased urine output in patients with less severe acute kidney injury, which may have been due to increased intravascular volume or through a diuretic effect. ${ }^{19}$ Conversely, serum creatinine levels were consistently higher in the HES group, suggesting a progressive reduction in creatinine clearance and more severe acute kidney injury.

The use of HES was associated with the administration of lower volumes of resuscitation fluid, although the ratio between HES and saline was similar to that observed in other blinded trials, suggesting that the use of HES was not associated with a substantive volume-sparing effect. ${ }^{8,20,21}$

The use of HES was also associated with increased use of blood products and a decrease in the rate of new cardiovascular failure; the latter, as defined by the SOFA score, indicates a reduction in the use of vasopressors, which was most likely due to increased intravascular expansion. However, this rate reduction was not associated with differences in other resuscitation end points, such as mean arterial pressure or serum lactate levels. Conversely, the use of HES was associated with new hepatic failure, which as defined by the SOFA score indicates an increase in serum bilirubin levels.

The use of HES was associated with an increased incidence of adverse events, particularly pruritus and rash. Our observations are consistent with studies that have shown increased tissue accumulation of HES within the reticuloendothelial system, which is manifested cutaneously as pruritus ${ }^{22}$ and may lead to acute kidney and hepatic injury. ${ }^{23,24}$

In studies involving patients with severe sepsis, HES was associated with increased mortality and acute kidney injury, resulting in the need for renal-replacement therapy. ${ }^{4,8}$ The patients in our study were at lower risk for death than the patients in these studies, but the point estimate for an increased relative risk of death and acute kidney injury associated with HES was consistent with the effects seen in these trials.

In conclusion, our study does not provide evidence that resuscitation with 6\% HES (130/0.4), as compared with saline, in the ICU provides any clinical benefit to the patient. Indeed, the use of HES resulted in an increased rate of renalreplacement therapy. Thus, the selection of resuscitation fluid in critically ill patients requires careful consideration of its safety, its potential effect on patient-centered outcomes, and its cost.

\footnotetext{
Supported by the National Health and Medical Research
} Council of Australia; the Ministry of Health, New South Wales Government, Australia; and Fresenius Kabi; and by a Practitioner Fellowship from the National Health and Medical Research Council of Australia (to Drs. Myburgh and Bellomo), by a Principal Research Fellowship from the National Health and Medical Research Council of Australia (to Dr. Cass), and by a Practitioner Fellowship from the Medical Research Foundation of the Royal Perth Hospital (to Dr. Webb).

Disclosure forms provided by the authors are available with the full text of this article at NEJM.org.

We thank all the patients and relatives who participated in this study and the doctors and nurses who cared for them. 
REFERENCES

1. Perel P, Roberts I. Colloids versus crystalloids for fluid resuscitation in critically ill patients. Cochrane Database Syst Rev 2012;6:CD000567.

2. Bunn F, Trivedi D. Colloid solutions for fluid resuscitation. Cochrane Database Syst Rev 2012;7:CD001319.

3. Finfer S, Liu B, Taylor C, et al. Resuscitation fluid use in critically ill adults: an international cross-sectional study in 391 intensive care units. Crit Care 2010;14(5): R185.

4. Brunkhorst FM, Engel C, Bloos F, et al Intensive insulin therapy and pentastarch resuscitation in severe sepsis. $\mathrm{N}$ Engl J Med 2008;358:125-39.

5. Schortgen F, Lacherade JC, Bruneel F, et al. Effects of hydroxyethylstarch and gelatin on renal function in severe sepsis: a multicentre randomised study. Lancet 2001;357:911-6.

6. Dart AB, Mutter TC, Ruth CA, Taback SP. Hydroxyethyl starch (HES) versus other fluid therapies: effects on kidney function. Cochrane Database Syst Rev 2010;1:CD007594.

7. Gattas DJ, Dan A, Myburgh J, Billot L, Lo S, Finfer S. Fluid resuscitation with 6\% hydroxyethyl starch (130/0.4) in acutely ill patients: an updated systematic review and meta-analysis. Anesth Analg 2012; 114:159-69.

8. Perner A, Haase N, Guttormsen AB, et al. Hydroxyethyl starch $130 / 0.42$ versus Ringer's acetate in severe sepsis. N Engl J Med 2012;367:124-34. [Erratum, N Engl J Med 2012;367:481.]

9. Crystalloid versus Hydroxyethyl Starch Trial (CHEST) Management Committee. The Crystalloid versus Hydroxyethyl Starch Trial: protocol for a multi-centre randomised controlled trial of fluid resusci- tation with $6 \%$ hydroxyethyl starch (130/0.4) compared to $0.9 \%$ sodium chloride (saline) in intensive care patients on mortality. Intensive Care Med 2011;37: 816-23.

10. Myburgh J, Li Q, Heritier S, Dan A, Glass P. Statistical analysis plan for the Crystalloid Versus Hydroxyethyl Starch Trial (CHEST). Crit Care Resusc 2012;14: 44-52. [Erratum, Crit Care Resusc 2012; 14:164.]

11. Kellum JA, Bellomo R, Ronco C. The concept of acute kidney injury and the RIFLE criteria. Contrib Nephrol 2007;156: 10-6.

12. Vincent JL, de Mendonça A, Cantraine $\mathrm{F}$, et al. Use of the SOFA score to assess the incidence of organ dysfunction/failure in intensive care units: results of a multicenter, prospective study. Crit Care Med 1998;26:1793-800.

13. Bone RC, Balk RA, Cerra FB, et al. Definitions for sepsis and organ failure and guidelines for the use of innovative therapies in sepsis. Chest 1992;101:164455.

14. Knaus WA, Draper EA, Wagner DP, Zimmerman JE. APACHE II: a severity of disease classification system. Crit Care Med 1985;13:818-29.

15. The SAFE Study Investigators. A comparison of albumin and saline for fluid resuscitation in the intensive care unit. N Engl J Med 2004;350:2247-56.

16. The NICE-SUGAR Study Investigators. Intensive versus conventional glucose control in critically ill patients. N Engl J Med 2009;360:1283-97.

17. Cohen J, Guyatt G, Bernard GR, et al. New strategies for clinical trials in patients with sepsis and septic shock. Crit Care Med 2001;29:880-6.
18. Ostermann M, Dickie H, Barrett NA. Renal replacement therapy in critically ill patients with acute kidney injury - when to start. Nephrol Dial Transplant 2012;27: 2242-8.

19. Jover JL, García JP, Martínez C, Espí A, Gregorí E, Almagro J. Hydroxyethyl starch to protect renal function in laparoscopic surgery. Rev Esp Anestesiol Reanim 2009; 56:27-30. (In Spanish.)

20. Guidet B, Martinet O, Boulain T, et al. Assessment of hemodynamic efficacy and safety of 6\% hydroxyethylstarch 130/0.4 vs. $0.9 \% \mathrm{NaCl}$ fluid replacement in patients with severe sepsis: the CRYSTMAS study. Crit Care 2012;16(3):R94.

21. James MF, Michell WL, Joubert IA, Nicol AJ, Navsaria PH, Gillespie RS. Resuscitation with hydroxyethyl starch improves renal function and lactate clearance in penetrating trauma in a randomized controlled study: the FIRST trial (Fluids in Resuscitation of Severe Trauma). Br J Anaesth 2011;107:693-702.

22. Kamann S, Flaig MJ, Korting HC. Hydroxyethyl starch-induced itch: relevance of light microscopic analysis of semi-thin sections and electron microscopy. J Dtsch Dermatol Ges 2007;5:204-8.

23. Dickenmann M, Oettl T, Mihatsch MJ. Osmotic nephrosis: acute kidney injury with accumulation of proximal tubular lysosomes due to administration of exogenous solutes. Am J Kidney Dis 2008;51: 491-503.

24. Christidis C, Mal F, Ramos J, et al. Worsening of hepatic dysfunction as a consequence of repeated hydroxyethylstarch infusions. J Hepatol 2001;35:726-32.

Copyright (c) 2012 Massachusetts Medical Society. 